\title{
Predictions on the transverse momentum spectra for charged particle production at LHC-energies from a two component model
}

\author{
A. A. Bylinkin ${ }^{1,2, a}$, N. S. Chernyavskaya ${ }^{1,2,3, b}$, A. A. Rostovtsev ${ }^{4, c}$ \\ ${ }^{1}$ Moscow Institute of Physics and Technology, MIPT, Moscow, Russia \\ ${ }^{2}$ National Research Nuclear University MEPhI, Moscow, Russia \\ ${ }^{3}$ Institute for Theoretical and Experimental Physics, ITEP, Moscow, Russia \\ ${ }^{4}$ Institute for Information Transmission Problems, IITP, Moscow, Russia
}

Received: 22 January 2015 / Accepted: 7 April 2015 / Published online: 25 April 2015

(C) The Author(s) 2015. This article is published with open access at Springerlink.com

\begin{abstract}
Transverse momentum spectra, $\mathrm{d}^{2} \sigma /\left(\mathrm{d} \eta \mathrm{d} p_{T}^{2}\right)$, of charged hadron production in $p p$-collisions are considered in terms of a recently introduced two component model. The shapes of the particle distributions vary as a function of the c.m.s. energy in the collision and the measured pseudorapidity interval. As a result the pseudorapidity of a secondary hadron in the moving proton rest frame is shown to be a universal parameter describing the shape of the spectra in $p p$-collisions. In order to extract predictions on the doubledifferential cross sections $\mathrm{d}^{2} \sigma /\left(\mathrm{d} \eta \mathrm{d} p_{T}^{2}\right)$ of hadron production for future LHC-measurements the different sets of available experimental data have been used in this study.
\end{abstract}

\section{Introduction}

Since the past decades a large volume of experimental data on charged hadron production spectra has been collected starting from the very first experiments performed on the Intersecting storage rings (ISR) at CERN till contemporary high statistics measurements carried out at the large hadron collider (LHC). It was shown [1] that the spectra of the produced hadrons are characterized by the exponential behavior for the low- $p_{T}$ part of the spectra, while the high- $p_{T}$ tail observes the power-like distribution typical for pQCD independently on the energy or type of the collision. However, these phenomena still lack theoretical explanation and can be described by various phenomenological models only. The most widely used model nowadays that combines exponential and power-like shapes is the Tsallis parameterization [2],

\footnotetext{
a e-mail: alexandr.bylinkin@ cern.ch

be-mail: nadezda.chernyavskaya@cern.ch

c e-mail: rostov@itep.ru
}

$\frac{\mathrm{d}^{2} \sigma}{\mathrm{d} \eta \mathrm{d} p_{T}^{2}}=\frac{A}{\left(1+\frac{E_{T}}{T \cdot N}\right)^{N}}$.

Indeed, in the low $p_{T} \rightarrow 0$ limit this function becomes similar to the Boltzmann exponent, while for high- $p_{T}$ it changes to a power-like shape. Recently a new qualitative model considering two sources of hadroproduction has been introduced [3,4], parameterizing the charged particle spectra by the sum of an exponential (Boltzmann-like) and a powerlaw $p_{T}$ distribution:

$\frac{\mathrm{d}^{2} \sigma}{\mathrm{d} \eta \mathrm{d} p_{T}^{2}}=A_{e} \exp \left(-E_{T k i n} / T_{e}\right)+\frac{A}{\left(1+\frac{p_{T}^{2}}{T^{2} \cdot N}\right)^{N}}$,

where $E_{T k i n}=\sqrt{p_{T}^{2}+M^{2}}-M$ with $M$ equal to the produced hadron mass. $A_{e}, A, T_{e}, T, N$ are free parameters, to be determined by a fit to the data. The detailed arguments for this particular choice are given in $[3,4]$. The exponential term in this model is associated with thermalized production of hadrons by valence quarks and a quark-gluon cloud coupled to them. The power-law term is related to the mini-jet fragmentation of the virtual partons (pomerons in $\mathrm{pQCD}$ ) exchanged between two colliding partonic systems.

A typical charged particle spectrum as a function of the transverse momentum fitted with this function (2) is shown in Fig. 1. As one can see, the exponential term dominates the particle spectrum at low $p_{T}$ values.

In $[3,4]$ it was also shown that this model allows one to get a much better description of the available experimental data than Tsallis. The comparison of these two parameterizations is also shown in Fig. 1. As one can see from the data/fit ratio, the Tsallis parameterization does not describe the details of the spectra resulting in "a roughly log-periodic behavior on top of the $q$-exponential one" [6]. Moreover, it was shown that the relative contributions of the two components of (2) to the spectra vary with the type of the collisions $[3,4](e q, p p, \gamma$ 


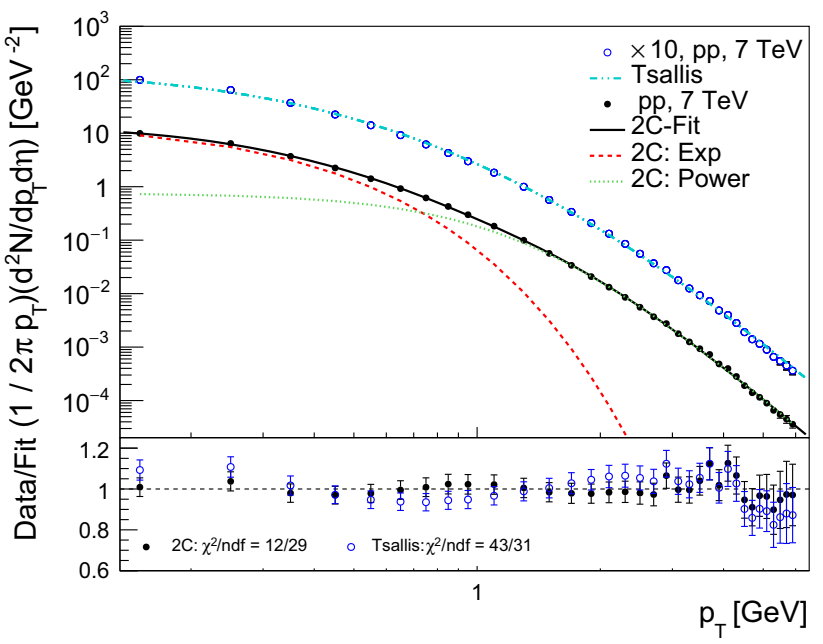

Fig. 1 Charged particle differential cross section $\left(1 / 2 \pi p_{T}\right) d^{2} \sigma /$ $d \eta d p_{T}$ [5] fitted to the two component (2C-fit) function (2) and Tsallis (1). The red (dashed) line shows the exponential term of the $2 \mathrm{C}$-fit and the green (dotted) line shows the power-law term, the blue (dashdot) line shows the Tsallis fit

$p, \gamma \gamma, A A)$, the type of the produced hadron [7], the charged multiplicity [8] and the measured pseudorapididty region [9], supporting the idea that the functional form (2) is not just a better parameterization due to the larger number of free parameters, but rather reflecting the underlying dynamics of hadroproduction.

Separating "soft" and "hard" contributions with this model allowed one to make the predictions on the mean $\left\langle p_{T}\right\rangle$ values as a function of multiplicity in the collision [8] and pseudorapidity distributions of charged particles [9]. However, the major interest of many studies in QCD is the transverse momentum spectrum itself. Therefore, in this article it is discussed how its shape varies in different experiments under various conditions. In $[3,4]$ it was shown that the parameters of the fit (2) show a strong dependence on the collision energy. Unfortunately, due to the fact that different collaborations measure charged particle production in their own phase space and under various experimental configurations, the dependences observed in $[3,4]$ were smeared and did not allow one to make strong predictions for further measurements. Thus, an approach to correct the measurements in order to allow for an accurate combination of different experimental data is proposed here.

\section{Parameter variations}

In [9] it was shown that two sources of hadroproduction described above contribute to different pseudorapidity regions: while the power-law term of (2) prevails in the midrapidity region $(\eta \sim 0)$, the exponential term dominates at

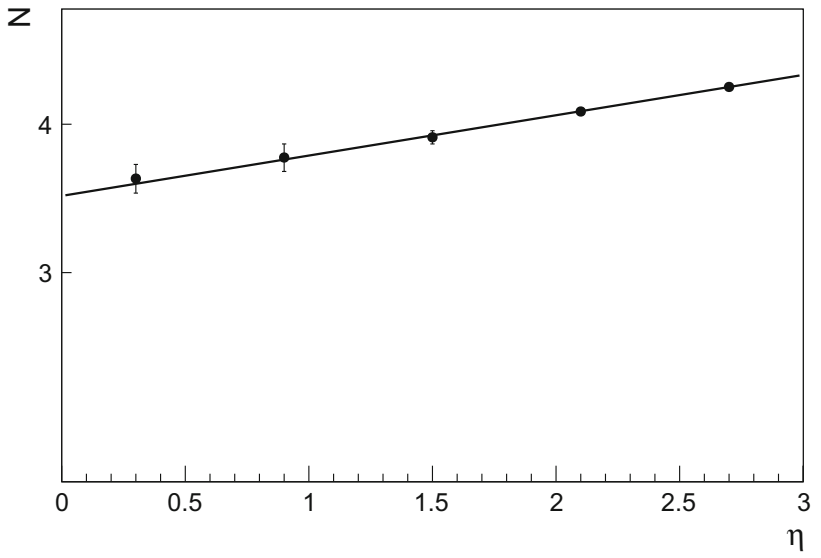

Fig. 2 Variation of the $N$ parameter of (2) obtained from the fits to the experimental data [11] as a function of pseudorapidity. The line shows a power-law fit of this variation

high values of $\eta$. Since each collaboration presents measurements on transverse momentum spectra in various pseudorapidity intervals, these variations might explain the smearing of the dependences in $[3,4]$. The idea to study parameter variations as a function of both collision energy and pseudorapidity region has already been successfully tested in [10].

To further study the variations of the spectra shapes as a function of pseudorapidity we use the data published by the UA1 experiment [11], which present charged particle spectra in five pseudorapidity bins, covering the total rapidity interval $|\eta|<3.0$. Figure $2^{1}$ shows how the parameter $N$ varies with the pseudorapidity together with a power-law fit of this variation. Note that the parameter shows a growth with pseudorapidity [9], which is explained by the higher thermalization of the spectra, as found in [9].

This variation can be parametrized in the following way:

$N=N^{0} \cdot\left(1+0.06 \cdot|\eta|^{1.52}\right)$

where $N^{0}$ denotes the parameter value at $\eta \sim 0$ and $\eta$ might be taken as a mean $\langle\eta\rangle$ of the measured pseudorapidity interval.

Since the variations of the parameter as a function of the pseudorapidity have been found, it is desirable to exclude its influence when studying the dependences of $N$ on the c.m.s. energy in a collision. This is possible if one combines only those data that have been measured in more or less the same pseudorapidity intervals. Hence, it is prudent to look at the combined data taken by the ISR [13], PHENIX [14] and ALICE [15] that were measured in the $|\eta|<0.8$ pseudorapidity region.

\footnotetext{
1 The error bars on this and further plots are obtained from the fits (2) to the experimental data (with statistical and systematic errors added in quadrature) performed in the ROOT framework [12].
} 


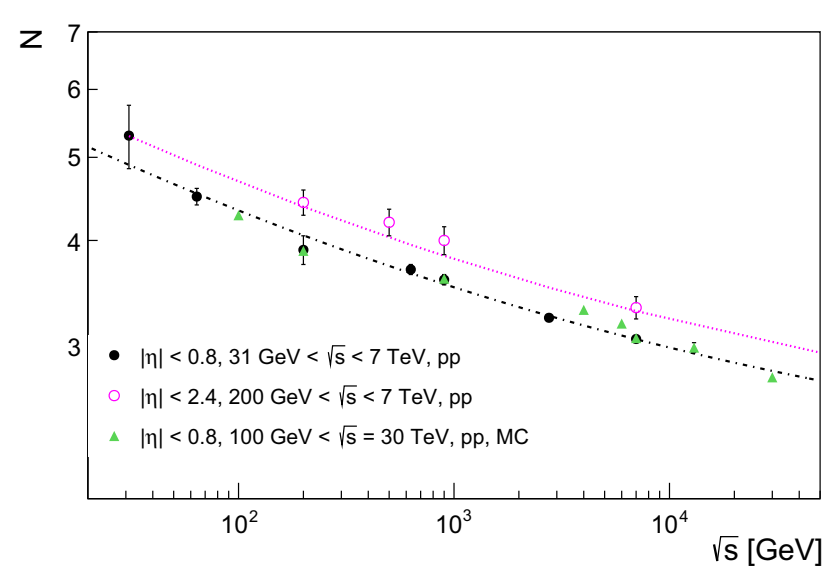

Fig. 3 Variation of the $N$ parameter of (2) obtained from the fits to the experimental data [13-15] and to the Monte Carlo generated data (full points) as a function of the c.m.s. energy $\sqrt{s}$ in a collision. The dash-dot line shows a fit of this variation. In addition, the open points show the parameters for the data measured in another pseudorapidity interval [5, 16] and the dotted line shows the predictions calculated according to (3)

Figure 3 shows the parameter $N$ variation as a function of the c.m.s. energy in a collision. One can notice that it can be characterized by the falling $N$-value. It is related to the fact that the probability to produce a high- $p_{T}$ mini-jet should grow with $\sqrt{s}$. Notably, this behavior correlates with the fact that $N$ decreases when the rapidity interval between the secondary hadron and the initial proton increases.

One can extract the following parametrization from the fit shown in Fig. $3^{2}$ :

$N=2.01+5.46 \cdot s^{-0.093}$

Remarkably, in the $s \rightarrow \infty$ limit $N \rightarrow 2$, which corresponds at large $p_{T}$ to $\mathrm{d} \sigma / \mathrm{d} p_{T}^{2} \propto 1 / p_{T}^{4}$, such a behavior is expected in pQCD where the elementary parton-parton cross section $\mathrm{d} \hat{\sigma} / \mathrm{d} p_{T}^{2} \propto \alpha_{S}^{2} / p_{T}^{4}$, that is, up to the logarithms $\mathrm{d} \sigma / \mathrm{d} p_{T}^{2} \propto 1 / p_{T}^{4}$. However, in a real collision one should take the initial conditions and the kinematic restrictions into account, resulting in a higher value of $N$ for lower $\sqrt{s}$. Obviously, such initial conditions should become negligible in the $s \rightarrow \infty$ limit, which is confirmed by the observed behavior (4).

In addition, Fig. 3 shows UA1 [16] and CMS [5] data measured under different experimental conditions. In these measurements the pseudorapidity interval was much wider $(|\eta|<2.5)$ than in [13-15]. Therefore, one can compare the parameter values obtained from the fit of these data (open points in Fig. 3) to the values extrapolated from (3) with $N^{0}$ calculated according to (4) and $|\eta|=1.25$ (pointed lines) and see a rather good agreement. Notably, this behavior correlates with the fact that $N$ decreases when the rapidity inter-

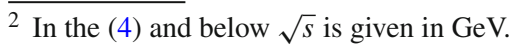

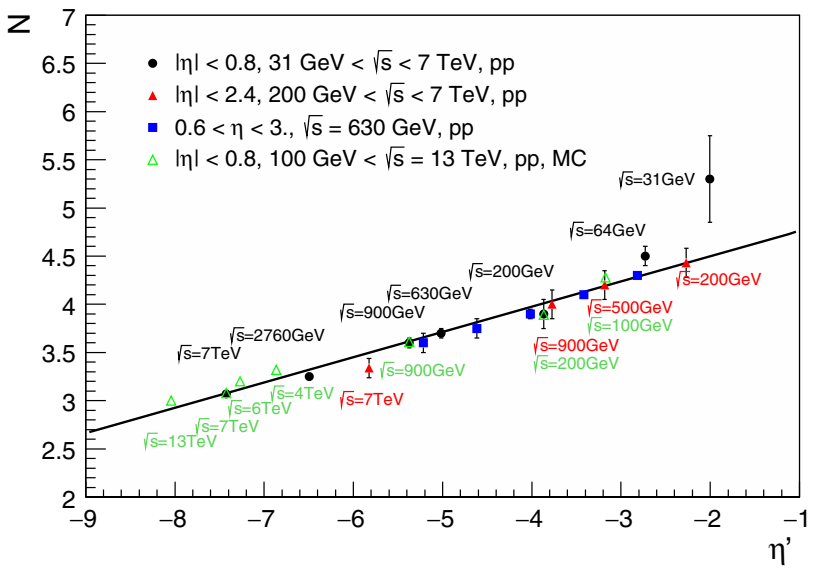

Fig. 4 The dependence of the $N$ parameter on the pseudorapidity of the secondary hadron in the moving proton rest frame. The data points from different experiments $[5,11,13-16]$ are shown together with the generated data (MC). The line shows the linear fit as a function of $\eta^{\prime}$ expressed by (6)

val between the secondary hadron and the same side beam proton increases.

Let us now check this correlation explicitly and calculate the rapidity interval in the moving proton rest frame according to a simple formula:

$\eta^{\prime}=|\eta|-\log \left(\sqrt{s} / 2 m_{p}\right)$

where $m_{p}$ is the mass of the incoming proton. The results of this procedure are shown in Fig. 4. Surprisingly, all the points came to a single line in this interpretation. To understand the origin of this universality one might use Monte Carlo (MC) generators: hard processes at large $p_{T}$ are known to be described by MC generators pretty well, thus it is expected to get the value of the $N$-parameter from the fits of the $\mathrm{MC}$ generated spectra rather close to the real data, but with a higher accuracy and in a wider collision energy range. To check this universality, we have produced the Monte Carlo samples for proton-proton collisions at different energies for inelastic (INEL) events with the PYTHIA 8.2 generator [17]. Indeed, the values of the parameter $\mathrm{N}$ extracted from the fits to the MC-generated spectra are nicely placed at the same line. Thus, a universal parameter describing the shape of the transverse momentum spectra in $p p$-collisions has been found.

$N=5.04+0.27 \eta^{\prime}$

A further check of the observed phenomena can be made if one plots $N$ as a function of the logarithm of the maximal kinematically allowed transverse momentum $p_{T_{\max }}$ of the secondary hadron at the specific pseudorapidity $\eta$.

$p_{T_{\max }}=\frac{\sqrt{s}}{2} \sin \left[2 \cdot \tan ^{-1}(\exp (-|\eta|))\right]$ 


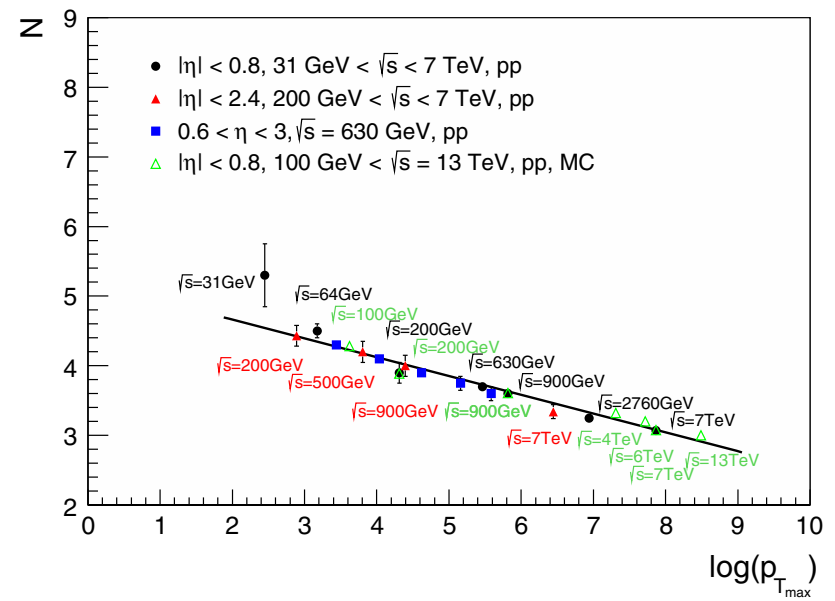

Fig. 5 The dependence of the $N$ parameter on the maximal kinematically allowed transverse momentum of the secondary hadron. The data points from different experiments $[5,11,13-16]$ are shown together with the generated data (MC). The line shows the linear fit as a function of $p_{T_{\max }}$

This dependence is shown in Fig. 5. One can see that larger $N$ corresponds to smaller $p_{T_{\max }}$. That should correspond to the $x \rightarrow 1$ limit of PDFs, where the decrease of the perturbative cross section is modified by the fall-off of the parton distribution functions (PDFs).

Remarkably, similarly to $N$, the $T$ and $T_{e}$ also show dependences as a function of both the collision energy $\sqrt{s}$ and the measured pseudorapidity interval $\eta$. The variations of the $T$ and $T_{e}$ parameters were studied in [10]. In [10] the possible theoretical explanation of the thermalized particle production was presented and the following proportionalities were established:

$T=409 \cdot(\sqrt{s})^{0.06} \cdot \exp (0.06|\eta|) \mathrm{MeV}$,

$T_{e}=98 \cdot(\sqrt{s})^{0.06} \cdot \exp (0.06|\eta|) \mathrm{MeV}$.

The parametrizations for $T$ and $T_{e}$ differ only by a constant factor. However, both the $T$ and the $T_{e}$ parameters reflect the thermalization which is stronger at higher energies and when closer to the valence quarks. Therefore, the (8) and (9) parametrizations which are functions of the center of mass energy and rapidity interval can be rewritten in a form with only one universal parameter. This universal parameter is the rapidity distance $\eta^{\prime \prime}$ from the farther incoming proton.

$\eta^{\prime \prime}=|\eta|+\log \left(\sqrt{s} / 2 m_{p}\right)$

Using $(8,9)$ we get the universal dependence ${ }^{3}$ :

$T=409 \cdot \exp \left(0.06 \eta^{\prime \prime}\right) \cdot\left(2 m_{p}\right)^{0.06} \mathrm{MeV}$,

$T=98 \cdot \exp \left(0.06 \eta^{\prime \prime}\right) \cdot\left(2 m_{p}\right)^{0.06} \mathrm{MeV}$.

The dependences are shown on Fig. 6.

\footnotetext{
${ }^{3}$ In (11), (12), and (23) $m_{p}$ is given in units of $1 \mathrm{GeV} / \mathrm{c}^{2}$.
}

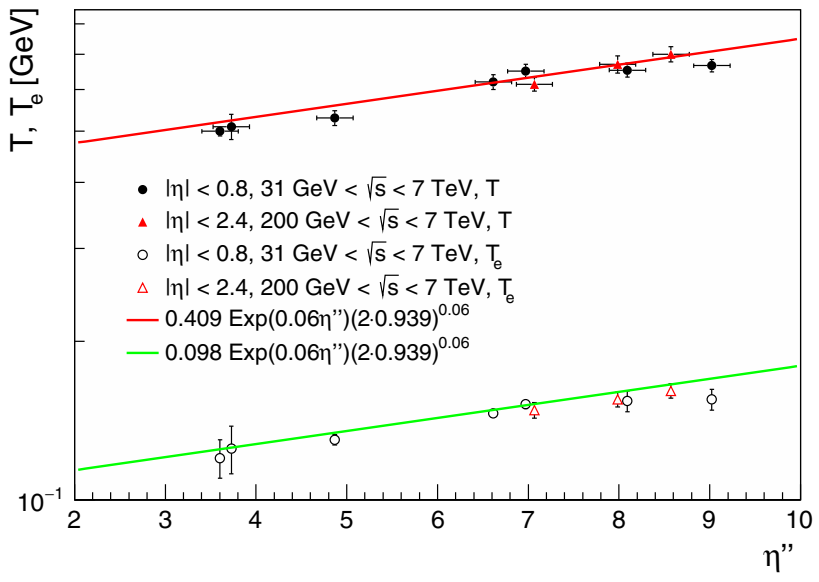

Fig. 6 The dependence of the $T$ and $T_{e}$ parameters on the pseudorapidity of the secondary hadron in the moving opposite side proton rest frame. The data points from different experiments [5,11,13-16] are shown. The lines show the dependences (11)-(12), respectively

\section{Prediction for further measurements}

Though MC was shown to provide a nice description of the high-pt part of the spectra, the nature of the soft particle production still remains ambiguous and varies for different MC generators. Therefore, the next step in understanding the underlying dynamics of high energy hadronic processes was done in a recent analysis [10].

In [9] it was shown that the introduced approach is able to give predictions on the pseudorapidity distributions in high energy collisions for non-single diffractive events (NSD). Using the parameterizations from [9] in addition with (3)(9) one can provide a formula that describes the shapes of charged particle spectra, being a function only of the center of mass energy and a measured pseudorapidity region. Let us now summarize all the equations to obtain the final result. ${ }^{4}$

$$
\begin{aligned}
\left(\frac{\mathrm{d} \sigma}{\mathrm{d} \eta}\right)_{\text {power }}= & A_{\text {power }} \cdot \exp \left(-\frac{\eta^{2}}{2 \sigma_{\text {power }}^{2}}\right), \\
\left(\frac{\mathrm{d} \sigma}{\mathrm{d} \eta}\right)_{\exp }= & A_{\exp } \cdot \exp \left(-\frac{\left(\eta-\eta_{\exp }\right)^{2}}{2 \sigma_{\exp }^{2}}\right) \\
& +A_{\exp } \cdot \exp \left(-\frac{\left(\eta+\eta_{\exp }\right)^{2}}{2 \sigma_{\exp }^{2}}\right),
\end{aligned}
$$

$\sigma_{\text {power }}=0.217+0.235 \cdot \ln \sqrt{s}$,

$\eta_{\exp }=0.692+0.293 \cdot \ln \sqrt{s}$,

$\sigma_{\text {exp }}=0.896+0.136 \cdot \ln \sqrt{s}$,

$A_{\text {power }}=0.13 \cdot s^{0.175}$

$A_{\text {exp }}=0.76 \cdot s^{0.106}$.

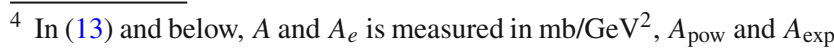
in $\mathrm{mb}$.
} 
Now, with the knowledge of the variations of the $T, N$, and $T_{e}$ parameters and the exponential and power-law contributions one can calculate the normalization parameters $A$ and $A_{e}$ in (2) in the following way:

$$
\begin{aligned}
\frac{\mathrm{d} \sigma}{\mathrm{d} \eta}=\left(\frac{\mathrm{d} \sigma}{\mathrm{d} \eta}\right)_{\exp }+\left(\frac{\mathrm{d} \sigma}{\mathrm{d} \eta}\right)_{\text {pow }}=\int_{0}^{\infty} \frac{\mathrm{d}^{2} \sigma}{\mathrm{d} \eta \mathrm{d} P_{T}^{2}} \mathrm{~d} p_{T}^{2}, \\
\left(\frac{\mathrm{d} \sigma}{\mathrm{d} \eta}\right)_{\text {pow }}=\int_{0}^{\infty} \frac{A}{\left(1+\frac{p_{T}^{2}}{T^{2} \cdot N}\right)^{N}} \mathrm{~d} p_{T}^{2}=\frac{A N T^{2}}{N-1}, \\
\left(\frac{\mathrm{d} \sigma}{\mathrm{d} \eta}\right)_{\exp }=\int_{0}^{\infty} A_{e} \exp \left(-E_{T k i n} / T_{e}\right) \mathrm{d} p_{T}^{2} \\
=2 A_{e} T_{e}\left(m+T_{e}\right) .
\end{aligned}
$$

Thus, we get the set of equations allowing us to make a prediction for a double-differential cross section, using the formula (2):

$$
\left\{\begin{array}{l}
|\eta|=\left(|\eta|_{\max }+|\eta|_{\min }\right) / 2 \\
N=5.04+0.27 \eta^{\prime} \\
T=409 \cdot \exp \left(0.06 \eta^{\prime \prime}\right) \cdot\left(2 m_{p}\right)^{0.06} \\
T=98 \cdot \exp \left(0.06 \eta^{\prime \prime}\right) \cdot\left(2 m_{p}\right)^{0.06} \\
A_{e}=\frac{1}{2 T_{e}\left(m+T_{e}\right)}(\mathrm{d} \sigma / \mathrm{d} \eta)_{\exp } \\
A=\frac{(N-1)}{N T^{2}}(\mathrm{~d} \sigma / \mathrm{d} \eta)_{\mathrm{pow}},
\end{array}\right.
$$

where $\eta^{\prime}$ and $\eta^{\prime \prime}$ can be calculated using (5) and (10) correspondingly.

Now, one can calculate double-differential cross sections $\mathrm{d}^{2} \sigma /\left(\mathrm{d} \eta \mathrm{d} p_{T}^{2}\right)$ of charged particle production in high energy collisions at different energies for NSD events. These predictions are shown in Fig. 7 for $|\eta|<0.8$ and the $|\eta|<2.4$ pseudorapidity intervals together with the experimental data measured by ALICE [18] and CMS [5]. A good agreement of the prediction with the data can be observed. Thus, the

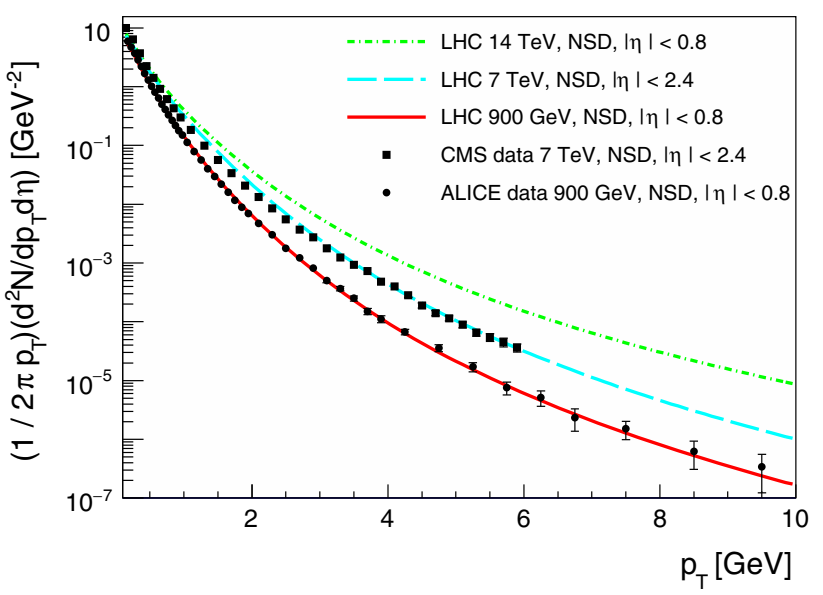

Fig. 7 Predictions of the yield of charged particles $\left(1 / 2 \pi p_{T}\right) \mathrm{d}^{2} N /\left(\mathrm{d} \eta \mathrm{d} p_{T}\right)$ in high energy collisions in NSD events together with data points from the ALICE [18] and CMS [5] experiments results (23) give us a powerful tool for predicting the spectral shapes in NSD events.

\section{Conclusion}

In conclusion, transverse momentum spectra in $p p$-collisions have been considered using a two component model. Variations of the parameters obtained from the fit have been studied as a function of the pseudorapidity $\eta$ and the c.m.s. energy $\sqrt{s}$ in the collision. A universal parameter describing a shape of the spectra in $p p$-collisions was found to be the pseudorapidity of a secondary hadron in the moving proton rest frame. Finally, the observed dependences, together with previous investigations allowed one to make predictions on double-differential spectra $\mathrm{d}^{2} \sigma /\left(\mathrm{d} p_{T}^{2} \mathrm{~d} \eta\right)$ at higher energies, successfully tested on the available experimental data.

Acknowledgments The authors thank Prof. Mikhail Ryskin, Prof. Torbjorn Sjostrand, and Dr. Peter Skands for fruitful discussions and help provided during the preparation of this paper. A. Rostovtsev is grateful to the Russian Foundation for Sciences for support (Project No. 14-50-00150).

Open Access This article is distributed under the terms of the Creative Commons Attribution 4.0 International License (http://creativecomm ons.org/licenses/by/4.0/), which permits unrestricted use, distribution, and reproduction in any medium, provided you give appropriate credit to the original author(s) and the source, provide a link to the Creative Commons license, and indicate if changes were made.

Funded by SCOAP ${ }^{3}$.

\section{References}

1. R. Hagedorn, Riv. Nuovo Cim. 6N10, 1 (1983)

2. C. Tsallis, Braz. J. Phys. 29, 1 (1999)

3. A.A. Bylinkin, A.A. Rostovtsev, Phys. Atom. Nucl. 75, 999 (2012) [Yad. Fiz. 75, 1060 (2012)]

4. A.A. Bylinkin, A.A. Rostovtsev, in ISMD 2010 proceedings, pp. 103-108. doi:10.5689/UA-PROC-2010-09/14. arXiv:1008.0332 [hep-ph]

5. V. Khachatryan et al. [CMS Collaboration], Phys. Rev. Lett. 105, 022002 (2010). arXiv:1005.3299 [hep-ex]

6. C.Y. Wong, G. Wilk, L.J.L. Cirto, C. Tsallis, EPJ Web Conf. 90, 04002 (2015). arXiv:1412.0474 [hep-ph]

7. A.A. Bylinkin, A.A. Rostovtsev, Eur. Phys. J. C 74(5), 2898 (2014)

8. A.A. Bylinkin, M.G. Ryskin, Phys. Rev. D 90, 1, 017501 (2014). arXiv:1404.4739 [hep-ph]

9. A.A. Bylinkin, A.A. Rostovtsev, Nucl. Phys. B 888, 65 (2014). arXiv:1404.7302 [hep-ph]

10. A.A. Bylinkin, D.E. Kharzeev, A.A. Rostovtsev, Int. J. Mod. Phys. E 23, 0083 (2014). arXiv:1407.4087 [hep-ph]

11. G. Bocquet et al. [UA1 Collaboration], Phys. Lett. B 366, 434-440 (1996)

12. I. Antcheva, M. Ballintijn, B. Bellenot, M. Biskup, R. Brun, N. Buncic, P. Canal, D. Casadei et al., Comput. Phys. Commun. 180, 2499 (2009)

13. K. Alpgard et al. [UA5 Collaboration], Phys. Lett. B 112, 183 (1982)

14. A. Adare et al. [PHENIX Collaboration], Phys. Rev. C 83, 064903 (2011). arXiv:1102.0753 [nucl-ex] 
15. B.B. Abelev et al. [ALICE Collaboration], Eur. Phys. J. C 73, 2662 (2013). arXiv:1307.1093 [nucl-ex]

16. C. Albajar et al. [UA1 Collaboration], Nucl. Phys. B 335, 261 (1990)

17. T. Sjostrand, S. Ask, J.R. Christiansen, R. Corke, N. Desai, P. Ilten, S. Mrenna, S. Prestel, C.O. Rasmussen, P.Z. Skands, Comput. Phys. Commun. 191, 159-177 (2015). arXiv:1410.3012 [hep-ph]
18. K. Aamodt et al. [ALICE Collaboration], Phys. Lett. B 693, 53-68 (2010). arXiv:1007.0719 [hep-ex] 